\title{
A simplified account of cooperative effects in molecular walker teams
}

\author{
Darko Stefanovic \\ Department of Computer Science and Center for Biomedical Engineering \\ University of New Mexico \\ Albuquerque, New Mexico \\ darko@cs.unm.edu
}

\begin{abstract}
We study the motion of random walkers with residence time bias between first and subsequent visits to a site, as a model for synthetic molecular walkers based on catalytic DNA known as molecular spiders. Even though a single one-legged walker does not exhibit directional, superdiffusive motion, we find that a team of one-legged walkers on parallel tracks, connected by a flexible tether, does enjoy a superdiffusive transient.
\end{abstract}

\section{CCS CONCEPTS}

- Hardware $\rightarrow$ Emerging architectures;

\section{KEYWORDS}

molecular walkers, superdiffusion

\section{ACM Reference Format:}

Darko Stefanovic. 2018. A simplified account of cooperative effects in molecular walker teams. In NANOCOM '18: NANOCOM '18: ACM The Fifth Annual International Conference on Nanoscale Computing and Communication, September 5-7, 2018, Reykjavik, Iceland. ACM, New York, NY, USA, 2 pages. https://doi.org/10.1145/3233188.3233218

\section{INTRODUCTION}

Many of the metabolic, signalling, and control functions in eukaryotic cells are performed by molecular walkers carrying cargo along directional filaments or microtubules [5]; e.g., kinesin walkers are highly evolved, complex protein structures that exhibit a complex coordinated gait on a directed self-assembled track, powered by a supply of ATP in the surroundings [8,12]. Likewise, efficient guided transport of molecular cargo is an important goal in synthetic nanotechnology. Borrowing from nature, progress has been made on protein-based walkers. Designing proteins with desired function is a formidable challenge, and many groups have used DNA instead. A number of reported systems have relied on DNA strand displacement reactions to propel a walker strand along a self-assembled track, typically a directed track, and commonly built using the DNA origami technique [7]. The drive in these systems is the formation of additional Watson-Crick base pairs, and, in the directed case,

Permission to make digital or hard copies of all or part of this work for personal or classroom use is granted without fee provided that copies are not made or distributed for profit or commercial advantage and that copies bear this notice and the full citation on the first page. Copyrights for components of this work owned by others than ACM must be honored. Abstracting with credit is permitted. To copy otherwise, or republish, to post on servers or to redistribute to lists, requires prior specific permission and/or a fee. Request permissions from permissions@acm.org.

NANOCOM '18, September 5-7, 2018, Reykjavik, Iceland

(c) 2018 Association for Computing Machinery.

ACM ISBN 978-1-4503-5711-1/18/09..\$15.00

https://doi.org/10.1145/3233188.3233218 the essence of the system is a burnt-bridge mechanism-the walker cannot revisit a site.

We have been studying molecular spider systems [4], consisting of a streptavidin body and multiple single-stranded DNA "legs" that use catalytic DNA reactions, rather than DNA strand displacement. Each leg contains a DNAzyme sequence, whose catalytic action is to break a phosphodiester bond and thus cleave a substrate DNA sequence. The substrates can be displayed on sites in bulk, or on a surface track, such as a DNA origami tile. A leg can rebind to an already cleaved site, but rebinding is of much shorter duration than the first binding. Here the energy source for the walker is the track itself, but the mechanism is not burnt-bridge, and there is no inherent directionality in the track. Rather, directional motion is an emergent property that arises from a combination of a kinetic effect (residence time bias between first and subsequent visits) and of kinematic constraints (the geometry of the walker body restricting the gait). Experiments in bulk have shown that molecular spiders are chemically processive: when spiders and their substrates were randomly deposited in a matrix in a 1:3800 ratio, the spiders cleaved nearly all the available substrates before dissociating from the matrix [4]. The concomitant release of products is nearly complete and happens at an essentially constant rate [11], which holds promise in biomedical applications. Experiments on DNA origami have shown that molecular spiders can follow patterned nanoscale tracks [2]. While we await experimental confirmation of the detailed motion, a number of mathematical modelling studies $[1,6,9,10]$ have characterized the spiders' walking gaits using coarse-grained stochastic models, up to predicting their ability to do organized mechanical work against external force [3]. The foremost question in such guided transport, for its application in signalling, is whether the motion is ordinary diffusive or superdiffusive. Consider the walker's displacement from a starting position: if the mean squared displacement scales linearly with time, the motion is diffusive; if it scales as a power of time greater than 1, it is superdiffusive; in the extreme, scaling as the square of time, it is ballistic. Previous models have not suggested true superdiffusive behavior in molecular spider systems, and that possibility remains an intriguing question for future work; however, they have discovered exceptionally long superdiffusive transients, which would be relevant in experiments and applications. The mechanism of the transient superdiffusion was explained via the emergence of a boundary between the new and the previously visited sites, and the tendency of the multi-legged spider to cling to this boundary, provided there is a residence time bias $r$ between the first and the subsequent visits to a site [10]. Detailed results were obtained for a two-legged spider with hand-over-hand gait in one dimension [10]. The key insight is that the spider alternates 
between being on the visited/new boundary (and moving forward ballistically) and drifting in the sea of previously visited sites; each period this sea becomes bigger, hence the eventual breakdown of superdiffusion.

Approaches for improving the transient and perhaps asymptotic behavior of molecular spiders include modified chemistry, different walker body and leg geometry, and the use of walker teams. Walker teams are inspired by nature's molecular motor teams [13]. Rank et al. [6] provided a detailed analysis of teams of two-legged molecular spiders, on parallel one-dimensional tracks, connected by a "leash", i.e., a kinematic constraint that no two spiders can be more than a certain distance apart. They showed that teams of two, three, and four spiders successively outperform a single spider, for a range of leash lengths and chemical kinetics.

\section{RESULTS}

Here we ask: can we separate the effects of having a team of walkers from the effects of each walker having multiple legs? Our model system uses single-legged walkers, each on its own one-dimensional track, connected by a leash. Each track is prepared with fresh substrates for $x \geq 0$, and consumed products for $x<0$; walkers start at $x=0$. With a kinetic Monte Carlo simulation we obtained 60,000 trajectories each for $n=1,2,3,4$ walkers, setting the effective leash length to $d=8$ for a direct comparison with the results of [6]. As in that work, the unit of length is the lattice spacing, and the unit of time is such that the hopping rate from visited sites is 1 , from new sites $r<1$. In Figure 1 we see that a single single-legged walker is not directionally biased-this is to be expected, and is crucially different from the single two-legged walker in [6]. As soon as multiple walkers are leashed to form a team, the team is directionally biased in the $+x$ direction, and at moderate times such as $t=10^{6}$ the position of the team is fairly narrowly distributed for team size 4. These results parallel those of [6]. In Figure 2, teams with more walkers exhibit more marked superdiffusive transients, which, however, decay to ordinary diffusion by time $10^{8}$.

In summary, we observe that the essential effects of a walker team are present even when each single team member is a singlelegged walker rather than a two-legged spider. In addition to the numerical simulation results here, we will carry out a mathematical analysis of the team, which in turn will be simplified by having simple teammates. Furthermore, we will study such teams when placed not on separate tracks but on a shared track, modelling a microtubule. Intuitively, the geometric constraint of the leash unites the multiple walkers into a soft-bodied walker with multiple legs.

\section{ACKNOWLEDGMENTS}

This material is based upon work supported by the National Science Foundation under grant CCF-1422840.

\section{REFERENCES}

[1] Tibor Antal and P. L. Krapivsky. 2007. Molecular spiders with memory. Physical Review E 76, 021121 (2007)

[2] Kyle Lund, Anthony J. Manzo, Nadine Dabby, Nicole Michelotti, Alexander Johnson-Buck, Jeanette Nangreave, Steven Taylor, Renjun Pei, Milan N. Stojanovic, Nils G. Walter, Erik Winfree, and Hao Yan. 2010. Molecular robots guided by presciptive landscapes. Nature 465, 7295 (2010).

[3] Mark J. Olah and Darko Sefanovic. 2013. Superdiffusive transport by multivalent molecular walkers moving under load. Physical Review E 87, 6 (2013).

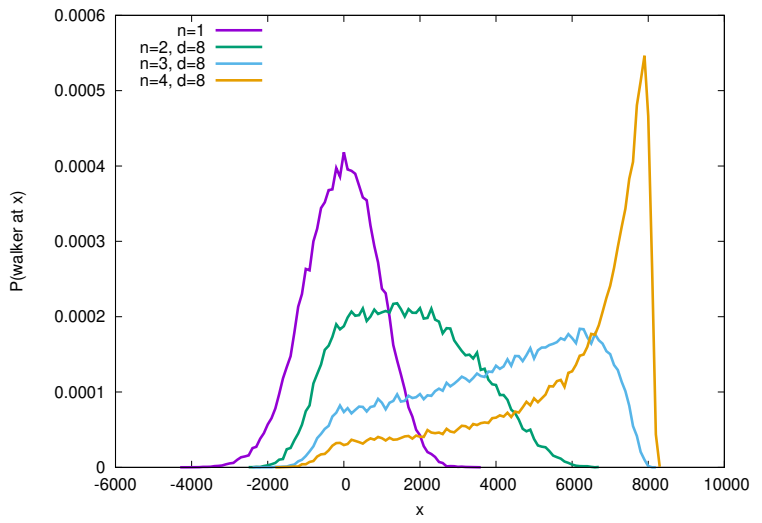

Figure 1: Position probability distribution, for residence time bias $r=0.01$, at time $t=10^{6}$.

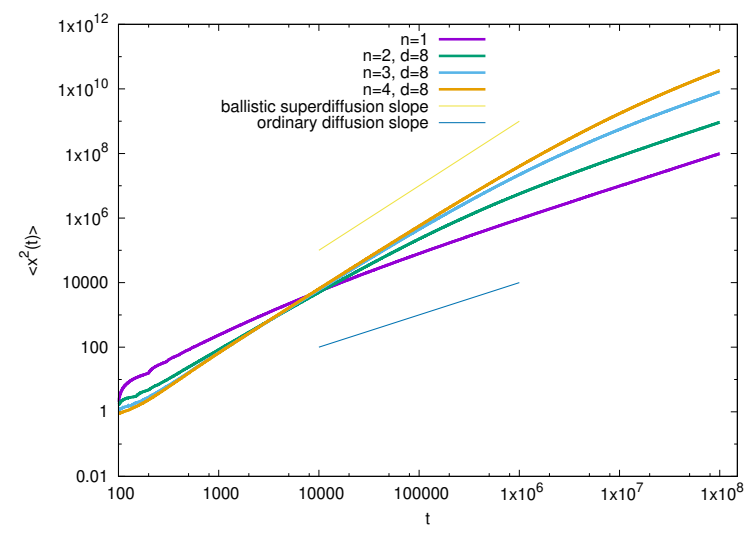

Figure 2: Mean squared displacement as a function of time, for residence time bias $r=0.01$.

[4] Renjun Pei, Steven K. Taylor, Darko Stefanovic, Sergei Rudchenko, Tiffany E. Mitchell, and Milan N. Stojanovic. 2006. Behavior of Polycatalytic Assemblies in a Substrate-Displaying Matrix. Fournal of the American Chemical Society 128, 39 (2006).

[5] Rob Phillips, Jane Kondev, Julie Theriot, and Hernan G. Garcia. 2013. Physical Biology of the Cell (2nd ed.). Garland Science, London and New York.

[6] Matthias Rank, Louis Reese, and Erwin Frey. 2013. Cooperative effects enhance the transport properties of molecular spider teams. Physical Review E 87, 3 (2013), 032706.

[7] Paul W. K. Rothemund. 2006. Folding DNA to create nanoscale shapes and patterns. Nature 440 (2006), 297-302.

[8] Manfred Schliwa and Gunther Woehlke. 2003. Molecular Motors. Nature 422, 6933 (2003), 759-765.

[9] Oleg Semenov, David Mohr, and Darko Stefanovic. 2013. First Passage Properties of Molecular Spiders. Physical Review E 88, 012724 (2013).

[10] Oleg Semenov, Mark J. Olah, and Darko Stefanovic. 2011. Mechanism of Diffusive Transport in Molecular Spider Models. Physical Review E 83, 021117 (2011).

[11] Darko Stefanovic, Milan N. Stojanovic, Mark J. Olah, and Oleg Semenov. 2013. Catalytic Molecular Walkers: Aspects of Product Release. In 12th European Conference on Artificial Life.

[12] Ronald D. Vale and Ronald A. Milligan. 2000. The Way Things Move: Looking Under the Hood of Molecular Motor Proteins. Science 288, 5463 (2000), 88-95.

[13] Andrej Vilfan, Erwin Frey, Franz Schwabl, Manfred Thormählen, Young-Hwa Song, and Eckhard Mandelkow. 2001. Dynamics and cooperativity of microtubule decoration by the motor protein kinesin. Fournal of Molecular Biology 312, 5 (2001), $1011-1026$ 